\title{
Analysis of magnolol and honokiol in biological fluids by capillary zone electrophoresis
}

\author{
Chiu-Ling Chen ${ }^{\text {a,b }}$, Po-Ling Chang ${ }^{\text {b }}$, Shoei-Sheng Lee ${ }^{a}$, Fu-Chuo Peng ${ }^{d}$, \\ Ching-Hua Kuo ${ }^{\mathrm{a}, *}$, Huan-Tsung Chang ${ }^{\mathrm{b}, \mathrm{c}, * *}$ \\ a School of Pharmacy, College of Medicine, National Taiwan University, Taipei, Taiwan \\ ${ }^{\mathrm{b}}$ Department of Chemistry, National Taiwan University, Taipei, Taiwan \\ ${ }^{\mathrm{c}}$ Department of Natural Science Education, National Taitung University, Taitung, Taiwan \\ ${ }^{\mathrm{d}}$ Institute of Toxicology, College of Medicine, National Taiwan University, Taipei, Taiwan
}

Received 11 October 2006; received in revised form 22 December 2006; accepted 29 December 2006

Available online 10 January 2007

\begin{abstract}
Capillary zone electrophoresis (CZE) method was used for analysis of magnolol and honokiol. Under the optimized condition, CZE with UV absorption detection provided that the limit of detection was at $\mu \mathrm{M}$ level. To enhance detection sensitivity of magnolol and honokiol, CZE separation system was coupled with a laser-induced fluorescence (LIF) detector for the first time. The limits of detection of magnolol and honokiol were $12 \mathrm{nM}$ (3.20 $\mathrm{n} \mathrm{ml}^{-1}$ ) and $18 \mathrm{nM}\left(4.79 \mathrm{ng} \mathrm{ml}^{-1}\right.$ ), respectively, showing that the CZE-LIF system provides greater than 100-fold sensitivity improvements than does the CZE-UV system. The developed method was applied to analyze magnolol and honokiol in spiked human plasma samples, microsome incubation samples as a preliminary demonstration of its potential in pharmacokinetic studies.
\end{abstract}

(C) 2007 Elsevier B.V. All rights reserved.

Keywords: Magnolol; Honokiol; Capillary zone electrophoresis; Laser-induced fluorescence detection; Biological fluids

\section{Introduction}

The stem bark of Magnolia officinalis is one of the most important traditional herbal medicine in China and Japan for the treatment of thrombotic stroke, typhoid fever, depression, and headache [1,2]. Magnolol and honokiol were claimed to be the major pharmacologically active components in Magnolia officinalis. They possess activities against microbial [3], platelet aggregation [4], oxidation [5], anxiety [6], depression [7] and cancer [8]. Recent studies demonstrated that these compounds might be potential therapeutic agents for neurodegenerative diseases [9], acute promyelocytic leukemia [10] as well as

\footnotetext{
* Corresponding author at: School of Pharmacy, College of Medicine, National Taiwan University, No. 1, Section 1, Jen-Ai Road, Taipei 100, Taiwan. Tel.: +8862 23123456; fax: +886223919098.

** Corresponding author at: Department of Chemistry, National Taiwan University, No. 1, Section 4, Roosevelt Road, Taipei 106, Taiwan.

Tel.: +8862 33661171; fax: +886233661171

E-mail addresses: chkuo@ha.mc.ntu.edu.tw (C.-H. Kuo), changht@ntu.edu.tw (H.-T. Chang).
}

atherosclerosis [11]. In view of many in-progress researches associated with magnolol and honokiol, an efficient and sensitive analytical method is required to facilitate these studies.

Magnolol and honokiol in Magnolia officinalis, rats and human serum have been analyzed widely by HPLC methods [12-15]. Other studies also used supercritical fluid chromatography [16], high speed counter current chromatography [17] and liquid column chromatography-mass spectrometry [18] to quantitatively determine the magnolol and honokiol in Magnolia officinalis.

Capillary electrophoresis (CE) has been applied in the analysis of magnolol and honokiol in Magnolia officinalis [19-21]. However, because of a small injection volume and a short optical path-length of CE, low concentration sensitivity of CE-UV hampers its applicability to analyze magnolol and honokiol in biological fluids. The primary goal of our research was to develop a sensitive and efficient electrophoretic method to facilitate the pharmacokinetic study of magnolol and honokiol. To achieve this goal, both capillary zone electrophoresis (CZE) systems with UV absorption and laser-induced fluorescence (LIF) detection were developed to improve the sensitivity. We used 
spiked human plasma samples, microsome incubation samples to demonstrate the practicability of the methods developed in this study. To the best of our knowledge, this is the first time using CZE-LIF to analyze magnolol and honokiol in biological fluids.

\section{Experimental}

\subsection{Chemicals}

Magnolol and honokiol were purchased from Wako (Osaka, Japan). Disodium tetraborate was purchased from Merck (Darmstadt, Germany). Sodium hydroxide was purchased from Fluka (Buchs, Switzerland). NADPH was purchased from Sigma (St. Louis, MO, USA).

\subsection{Apparatus}

The CZE experiments with UV detection were carried out in a system consisting of a Prince programmable injector from Lauer Labs (Emmen, The Netherlands) and a $30 \mathrm{kV}$ high-voltage supply, connected to a UV-C absorbance detector from Dynamax (Rainin, Emeryville, CA, USA). A fused-silica capillary from Polymicro Technologies (Phoenix, AZ, USA) was used. The electropherograms were recorded using an EZChrom (Scientific Software, San Ramon, CA, USA) chromatographic data system.

For CZE experiments using LIF detection, the basic design of the separation system was previously described [22]. In brief, a high-voltage power supply (Gamma High Voltage Research, Ormond Beach, FL, USA) was used to drive electrophoresis. A diode-pumped solid state nanolaser (JDS Uniphase, Manteca, CA, USA) having a $5-\mathrm{mW}$ output at $266 \mathrm{~nm}$ was used to induce intrinsic fluorescence. The intrinsic fluorescence was collected using a $10 \times$ objective (numeric aperture 0.25) before reaching the photomultiplier tube (R928 from Hamamatsu, Shizuoka-Ken, Japan). The amplified current was transferred directly through a $10-\mathrm{k} \Omega$ resistor to a 24-bit A/D interface at $5 \mathrm{~Hz}$ (Borwin, JMBS Developments, Le Fontanil, France).

\subsection{Electrophoretic conditions}

CZE-UV experiments were performed on a $75 \mathrm{~cm}(60 \mathrm{~cm}$ effective length) $\times 50 \mu \mathrm{m}$ I.D. fused-silica capillary. CZE-LIF experiments were performed on a $40 \mathrm{~cm}(30 \mathrm{~cm}$ effective length) $\times 50 \mu \mathrm{m}$ I.D. fused-silica capillary. The new capillary was pre-conditioned prior to use by flushing with $1.0 \mathrm{M}$ sodium hydroxide, 0.2 M sodium hydroxide, de-ionized water, and running buffer each for $10 \mathrm{~min}$. At the beginning of each experiment, the capillary was washed with $0.2 \mathrm{M}$ sodium hydroxide, then followed by running buffer each for $5 \mathrm{~min}$.

The background electrolyte (BGE) solution was $45 \mathrm{mM}$ sodium tetraborate, $\mathrm{pH} 9.6$ (by titrating with $1 \mathrm{M} \mathrm{NaOH}$ ). Operation conditions for CZE-UV were set as follows: voltage, $30 \mathrm{kV}$; injection time, $4.8 \mathrm{~s}$ (hydrodynamic, $50 \mathrm{mbar}$ ); detection wavelength, $254 \mathrm{~nm}$. Operation conditions for CZE-LIF were set as follows: voltage, $12 \mathrm{kV}$; excitation wavelength, $266 \mathrm{~nm}$. The sample was hydrodynamically injected by raising the sample solution to create a $25 \mathrm{~cm}$ height difference relative to the buffer reservoir for $10 \mathrm{~s}$. All of the experiments were run at room temperature $\left(23 \pm 1{ }^{\circ} \mathrm{C}\right)$.

All the solutions were filtered through a $0.45 \mu \mathrm{m}$ filter (Millipore, Bedford, MA, USA) before use.

\subsection{Preparation of standard solution}

Stock solutions of magnolol and honokiol with concentrations of $5 \mathrm{mM}$ and $2 \mathrm{mM}$ were separately prepared in ethanol. These solutions were diluted with ethanol to give the working solution. The concentrations of magnolol and honokiol in the working solution were $1.25 \mathrm{mM}$ and $0.25 \mathrm{mM}$, respectively. Propyl paraben was added as an internal standard, and its final concentration was $0.2 \mathrm{mM}$.

\subsection{Sample preparation}

\subsubsection{Plasma sample}

Blood samples were picked up from healthy volunteers and immediately transferred to a heparinized microcentrifuge tube. Treatment of blood samples was according to the published method [14]. Magnolol, honokiol and internal standard were spiked to the blood sample then centrifuged at $3000 \mathrm{rpm}$ for $10 \mathrm{~min}$. The resulting plasma sample was then vortex-mixed with acetonitrile ( $\mathrm{ACN})$. After $15 \mathrm{~min}$, the mixture was centrifuged at $3000 \mathrm{rpm}$ for $5 \mathrm{~min}$ to remove proteins. The supernatant was then separately transferred into a $1.5-\mathrm{ml}$ tube containing $50-60 \mathrm{mg}$ sodium chloride. The suspension was vortex-mixed briefly and incubated at room temperature for $20 \mathrm{~min}$ and was vortex-mixed again. Finally, the plasma sample was centrifuged at $3000 \mathrm{rpm}$ for $5 \mathrm{~min}$. A portion of the supernatant was subjected to CZE-LIF analysis.

\subsubsection{Microsome incubation sample}

The incubation mixture (final volume $0.5 \mathrm{ml}$ ) contained $0.75 \mathrm{mg}$ rat liver microsomes, $0.2 \mathrm{mM}$ NADPH, $1.65 \mathrm{mM}$ $\mathrm{MgCl}_{2}, 0.1 \mathrm{M}$ sodium phosphate buffer, $\mathrm{pH} 7.4$, and $5 \mathrm{mM}$ magnolol, which was shaken ( 100 oscillations $\mathrm{min}^{-1}$ ) for $5 \mathrm{~min}$ at $37^{\circ} \mathrm{C}[23,24]$. Then the substrate was added to the mixture to start the reaction. After $30 \mathrm{~min}$ of incubation with shaking at $37^{\circ} \mathrm{C}$, the reaction was stopped by adding $0.2 \mathrm{ml}$ of methanol and the mixture was centrifuged at 39,000 rpm for $25 \mathrm{~min}$ at $4{ }^{\circ} \mathrm{C}$. Part of the supernatant $(100 \mu \mathrm{l})$ was mixed with $100 \mu \mathrm{l} \mathrm{ACN}$. After $15 \mathrm{~min}$, the mixture was centrifuged at $3000 \mathrm{rpm}$ for $5 \mathrm{~min}$ to separate precipitated proteins. The supernatant was then transferred into a $1.5-\mathrm{ml}$ tube containing $50-60 \mathrm{mg}$ sodium chloride. The suspension was vortex-mixed briefly and incubated at room temperature for $20 \mathrm{~min}$ and vortex-mixing again. Finally, the mixture was centrifuged at $3000 \mathrm{rpm}$ for $5 \mathrm{~min}$, and then was subjected to CZE-LIF analysis.

All sample solutions were filtered through a $0.45-\mu \mathrm{m}$ filter (Millipore, Bedford, MA, USA) before analysis. 


\section{Results and discussion}

\subsection{Method development}

Chemical structures of magnolol and honokiol are shown in Fig. 1. The $\mathrm{p} K_{\mathrm{a} 1}$ and $\mathrm{p} K_{\mathrm{a} 2}$ values of magnolol are 7.01 and 10.64, and those for honokiol are 9.92 and 10.71 [21]. Since their first $\mathrm{p} K_{\mathrm{a}}$ values are quite different, CZE method was used in this study. The $\mathrm{pH}$ values over the range of 8.0-10.0 of the solutions were chosen to preserve greater differences in the charged densities of magnolol and honokiol. When the $\mathrm{pH}$ value was below 8.8, honokiol remains in its neutral form and co-migrated with electroosmotic flow (EOF). With increasing the $\mathrm{pH}$ level, the degree of ionization increased and thus the peak efficiency increased as a result of reduced analyte adsorption. At $\mathrm{pH} 9.6$, the peak efficiency of honokiol was at its maximum. Further increases in the $\mathrm{pH}$ value encountered poor reproducibility and low peak efficiencies. Therefore, $\mathrm{pH} 9.6$ was considered as optimum separation $\mathrm{pH}$. Sodium tetraborate at concentrations between $30 \mathrm{mM}$ and $50 \mathrm{mM}$ with $\mathrm{pH} 9.6$ were tested in this study. Peak efficiency was found to increase with the increase of ionic strength. When sodium tetraborate was at the concentration higher than $50 \mathrm{mM}$, poor reproducibility occurred. Therefore, $45 \mathrm{mM}$ sodium tetraborate was chosen for the separation. Under the optimum separation conditions, the analysis was completed within $7 \mathrm{~min}$ as shown in Fig. 2.

\subsection{Method validation}

Relative standard deviation (RSD) of the repeatability $(n=10)$ and intermediate precision (inter-day, $n=3)$ of reten-<smiles>C=CCc1ccc(O)c(-c2ccc(O)c(CC=C)c2)c1</smiles>

Honokiol (1)<smiles>C=CCc1ccc(O)c(-c2cc(CC=C)ccc2O)c1</smiles>

Magnolol (2)<smiles>CCCOC(=O)c1ccc(O)cc1</smiles>

Propyl paraben (I.S.)

Fig. 1. Structures of honokiol, magnolol and propyl paraben (I.S.).

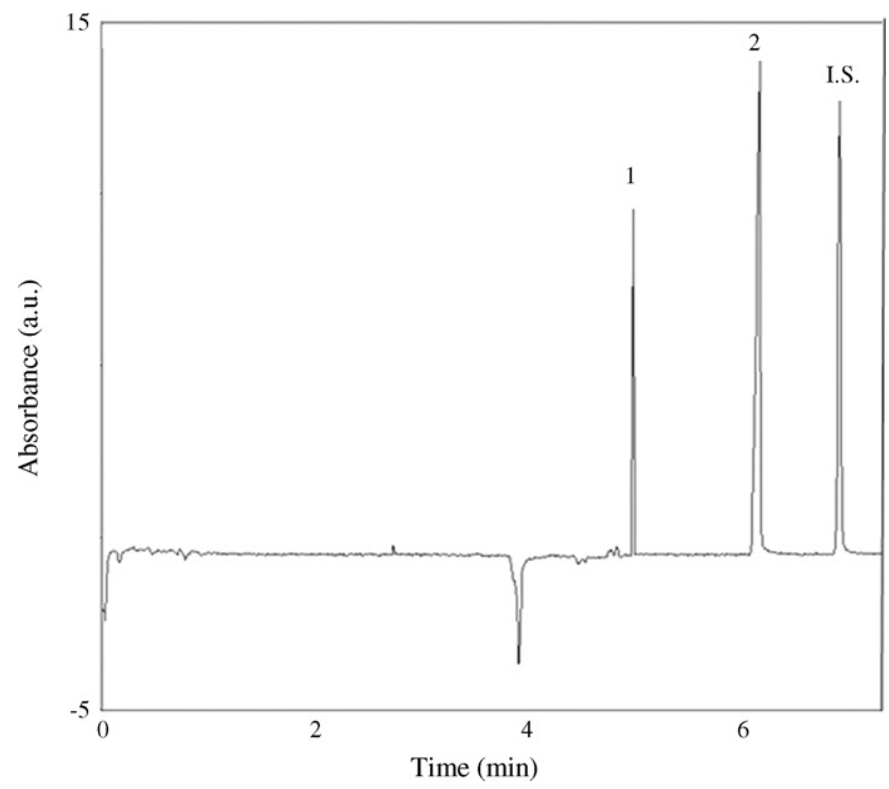

Fig. 2. Electropherogram of magnolol and honokiol under optimum separation conditions using UV detection. Separation conditions: fused-silica capillary, $75 \mathrm{~cm} \times 50 \mu \mathrm{m}$ I.D., $60 \mathrm{~cm}$ detection length; $45 \mathrm{mM}$ sodium tetraborate, $\mathrm{pH}$ 9.6; applied voltage, $30 \mathrm{kV}$; temperature, ambient $\left(23 \pm 1^{\circ} \mathrm{C}\right)$; injection, $50 \mathrm{mbar}$, 4.8 s. Peak: (1) honokiol; (2) magnolol.

tion times of magnolol and honokiol were within $1.51 \%$ and $1.94 \%$, respectively. The precision of peak area ratios of magnolol and honokiol to internal standard, propyl paraben, were tested at the $1.25 \mathrm{mM}$ and $0.25 \mathrm{mM}$. Repeatability $(n=10)$ and intermediate precision (inter-day, $n=3$ ) were within $2.76 \%$ and $6.46 \%$ RSD, respectively.

\subsubsection{Limit of detection}

When using a UV detector, the LODs of magnolol and honokiol were $4.26 \mu \mathrm{M}\left(1.13 \mu \mathrm{g} \mathrm{ml}^{-1}\right)$ and $3.75 \mu \mathrm{M}$ $\left(0.99 \mu \mathrm{g} \mathrm{ml}^{-1}\right)$. This LOD for magnolol is much higher than its concentration in rat plasma samples $\left(0.06-0.2 \mu \mathrm{g} \mathrm{ml}^{-1}\right)$ [14]. To enhance the sensitivity of the developed method, the CZE separation system was coupled with an LIF detector. The CZE-LIF system was expected to be suitable for the analysis of magnolol and honokiol, both possessing intrinsic fluorescence. When using CE-LIF, the analysis could be completed within $5 \mathrm{~min}$. The LODs of magnolol and honokiol were $12 \mathrm{nM}\left(3.20 \mathrm{ng} \mathrm{ml}^{-1}\right)$ and $18 \mathrm{nM}\left(4.79 \mathrm{ng} \mathrm{ml}^{-1}\right)$, respectively, showing that the CZE-LIF system provides more than 100 -fold sensitivity relative to the CZE-UV system. The LODs of magnolol and honokiol using CZE-LIF are much less than their plasma concentrations in rats, and thus it should be practical in pharmacokinetic study. To the best of our knowledge, the CE-LIF system provides the best sensitivity among standard CE methods for the analysis of magnolol and honokiol. Comparing to LC-MS/MS method [18], the present study provided comparable sensitivity and speed. However, the reagent consumption was greatly reduced with CZE-LIF method. Although the LODs of the present method were slightly higher than those obtained by micro-HPLC with electrochemical detection [25], the analytical time of CZE-LIF is greatly reduced ( $5 \mathrm{~min}$ versus $40 \mathrm{~min}$ ). 


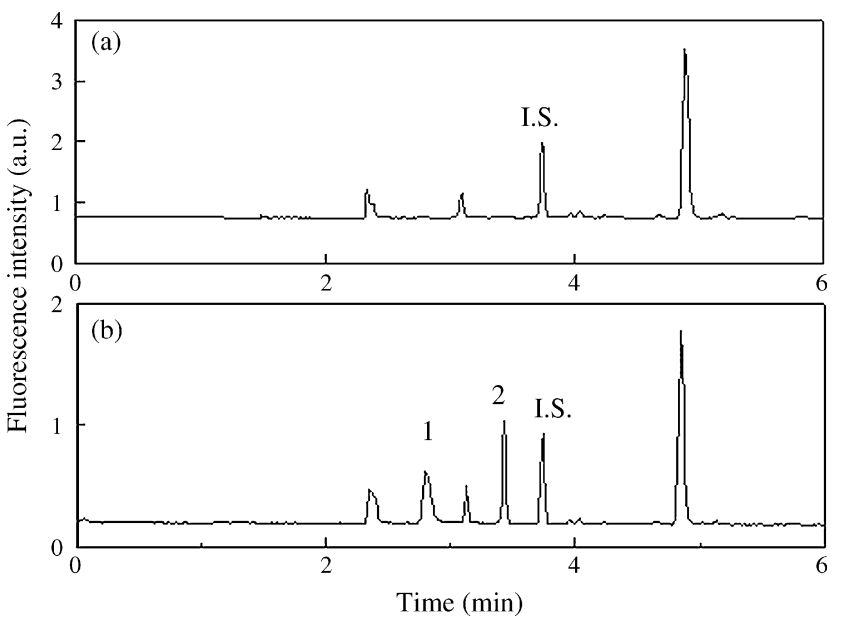

Fig. 3. Electropherograms of magnolol and honokiol in spiked human plasma. (a) blank human plasma; (b) human serum spiked with $100 \mathrm{ng} \mathrm{ml}^{-1}$ of magnolol and honokiol. Separation conditions: fused-silica capillary, $40 \mathrm{~cm} \times 50 \mu \mathrm{m}$ I.D., $30 \mathrm{~cm}$ detection length; $45 \mathrm{mM}$ sodium tetraborate, $\mathrm{pH}$ 9.6; applied voltage, $12 \mathrm{kV}$; temperature, ambient $\left(23 \pm 1{ }^{\circ} \mathrm{C}\right)$; injection, hydrodynamic injection, 10 s. Peak: (1) honokiol; (2) magnolol.

\subsection{Application}

Before applying CE-LIF method in the analysis of biological fluids, this method was subjected to method validation. The precision of peak area ratios were tested at $10 \mu \mathrm{M}$. Repeatability $(n=10)$ and intermediate precision (inter-day, $n=3)$ were within $2.35 \%$ and $3.18 \%$ RSD, respectively. Linearity of the CZE-LIF method was tested within the rage of $0.05-50 \mu \mathrm{M}$. The regression equations are $y=0.1183 x+0.8854\left(r^{2}=0.9933\right)$ for magnolol and $y=0.0305 x+0.0288$ for honokiol $\left(r^{2}=0.9984\right)$.

To demonstrate the potential application of CE-LIF in pharmacokinetic studies, the concentrations of magnolol and honokiol in spiked human plasma samples were determined. The concentrations $\left(0.1 \mu \mathrm{g} \mathrm{ml}^{-1}\right)$ of magnolol and honokiol were selected as average plasma concentration in pharmacokinetic

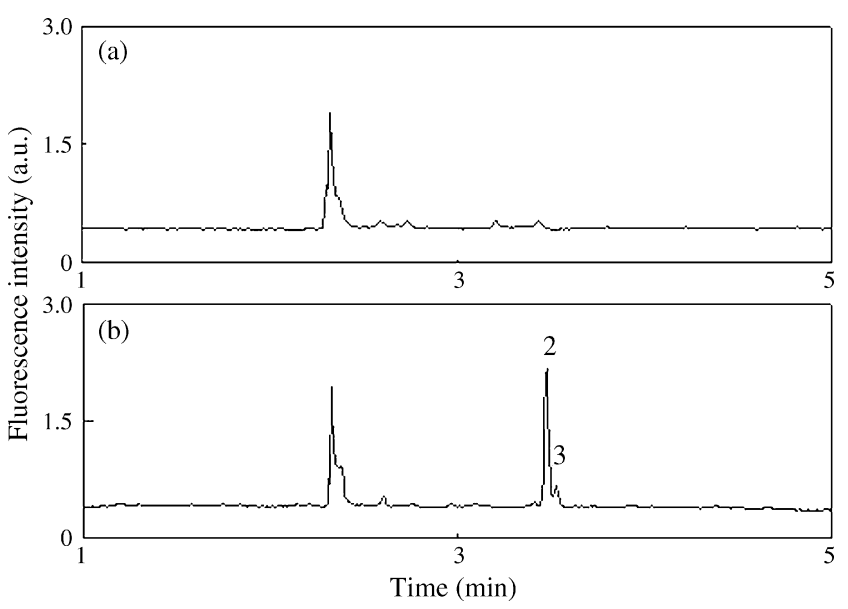

Fig. 4. Electropherograms of magnolol after incubation with rat liver microsome. (a) blank incubation medium; (b) $5 \mathrm{mM}$ magnolol incubated with rat liver microsome for $30 \mathrm{~min}$. Separation conditions: $45 \mathrm{mM}$ sodium tetraborate, $\mathrm{pH}$ 9.6; applied voltage, $12 \mathrm{kV}$; other conditions are as in Fig. 3. Peak: (2) magnolol; (3) unknown. study. The electropherograms of the human plasma sample and spiked human plasma sample are shown in Fig. 3a and b. Both magnolol and honokiol showed good signal intensity, and were well separated from sample matrix. The recoveries of magnolol and honokiol were $86.7 \%$ and $85.5 \%$, respectively, based on the peak area ratios for the two analytes.

Nakazawa et al. had performed the in vivo metabolism study of Magnolia officinalis [15]. In their study, they have found magnolol and its metabolite dihydroxydihydromagnolol in human urine samples. To demonstrate the potential of our developed method in studying metabolic pathway of magnolol, the microsome incubated samples were analyzed. A representative electropherogram is shown in Fig. 4. Magnolol was slightly overlapped with a small unknown peak, presumably its metabolite, although further study is essential to determine its chemical structure. Nevertheless, the developed method can still be used to quantitate magnolol after metabolizing by rat liver microsome.

\section{Conclusion}

Both CZE-UV and CZE-LIF methods for the analysis of magnolol and honokiol have been established in this study. With the ability to detect magnolol and honokiol in nanomolar level, the CZE-LIF method has great potential for pharmacokinetic and metabolism studies. Under optimal separation conditions, analysis with good peak efficiencies could be completed within $5 \mathrm{~min}$. The established method was successfully applied to analyze spiked human plasma sample and microsome incubated sample as the preliminary demonstration of its potential.

\section{Acknowledgement}

This study was supported by National Science Council of Taiwan (NSC 95-2320-B-002-113 and NSC 95-2113-M-002026-MY3).

\section{References}

[1] The Japanese Pharmacopoeia, The Ministry of Health, Labour and Welfare, Tokyo, 14th ed., 2001

[2] Committee of National Pharmacopoeia, Pharmacopoeia of People's Republic of China, vol.1, Press of Chemical Industry, Beijing, 2005, pp. 176.

[3] W.J. Syu, C.C. Shen, J.J. Lu, G.H. Lee, C.M. Sun, Chem. Biodiver. 1 (2004) 530.

[4] M.K. Pyo, Y.Y. Lee, H.S. Yun-Choi, Arch. Pharmacol. Res. 25 (2002) 325.

[5] H. Haraguchi, H. Ishikawa, N. Shirataki, A. Fukuda, J. Pharm. Pharmacol. 49 (1997) 209.

[6] H. Kuribara, E. Kishi, M. Kimura, S.T. Weintraub, Y. Maruyama, Pharmacol. Biochem. Behav. 67 (2000) 597.

[7] Y. Maruyama, H. Kuribara, M. Morita, M. Yuzurihara, S.T. Weintraub, J. Nat. Prod. 61 (1998) 135.

[8] H. Nagase, K. Ikeda, Y. Sakai, Planta Med. 67 (2001) 705.

[9] Y.R. Lin, H.H. Chen, C.H. Ko, M.H. Chan, Eur. J. Pharmacol. 537 (2006) 64.

[10] W.F. Fong, A.K.W. Tse, K.H. Poon, C. Wang, J. Biochem. Cell Biol. 37 (2005) 427.

[11] S.C. Chen, Y.L. Chang, D.L. Wang, J.J. Cheng, Br. J. Pharmacol. 148 (2006) 226.

[12] T.H. Tsai, C.F. Chen, J. Chromatogr. 598 (1992) 143. 
[13] T.H. Tsai, C.J. Chou, F.C. Cheng, C.F. Chen, J. Chromatogr. B 655 (1994) 41.

[14] X. Wu, X. Chen, Z. Hu, Talanta 59 (2003) 115.

[15] T. Nakazawa, T. Yasuda, K. Ohsawa, J. Pharm. Pharmacol. 55 (2003) 1583.

[16] K. Suto, Y. Ito, K. Sagara, H. Itokawa, J. Chromatogr. A 786 (1997) 366.

[17] X. Wang, Y.Q. Wang, Y.L. Geng, F.W. Li, C.C. Zheng, J. Chromatogr. A 1036 (2004) 171.

[18] Y.T. Wu, L.C. Lin, T.H. Tsai, Biomed. Chromatogr. 20 (2006) 1076.

[19] C.Y.C. Chou, T.H. Tsai, M.F. Lin, C.F. Chen, J. Liq. Chromatogr. Related Technol. 19 (1996) 1909.
[20] L. Liu, X. Wu, L. Fan, X. Chen, Z. Hu, Anal. Bioanal. Chem. 384 (2006) 1533.

[21] G. Chen, X. Xu, Y. Zhu, L. Zhang, P. Yang, J. Pharm. Biomed. Anal. 41 (2006) 1479

[22] Y.F. Huang, M.M. Hsieh, W.L. Tseng, H.T. Chang, J. Proteome Res. 5 (2006) 429

[23] F.C. Peng, C.C. Chang, C.Y. Yang, R.J. Edwards, J. Doehmerd, Toxicology 218 (2006) 172

[24] A.P. Alvares, G.J. Mannerin, Mol. Pharmacol. 6 (1970) 206.

[25] A. Kotani, S. Kojima, H. Hakamata, D. Jin, F. Kusu, Chem. Pharm. Bull. $53(2005) 319$. 